\title{
LHeC Accelerator Development
}

\section{Cruz-Alaniz E.*}

University of Liverpool and the Cockcroft Institute, UK

E-mail: e.cruz-alaniz@liverpool.ac.uk

Valloni A.

CERN, Switzerland

The Large Hadron electron Collider ( $\mathrm{LHeC})$ is a proposed facility which will exploit the Large Hadron Collider (LHC) beams for electron-proton/nucleus scattering, using a new $60 \mathrm{GeV}$ electron accelerator. Following the release of its detailed technical design report in 2012, the configuration of a linac with racetrack shape has been chosen for its default design. Further work has been pursued in order to adapt the electron and high luminosity optics and beam parameters, to achieve performance levels close to $10^{34} \mathrm{~cm}^{-2} \mathrm{~s}^{-1}$ as is desirable for precision Higgs physics with the LHeC. In parallel, work has focused on the design of an LHeC Test Facility (LTF) at CERN and to validate and prepare the Energy Recovery Linac (ERL) operation mode for the LHeC and to develop the required Superconducting (SC) RF technologies. The talk presents an overview on the design, recent activities and an outlook for further developments.

XXII. International Workshop on Deep-Inelastic Scattering and Related Subjects, 28 April - 2 May 2014

Warsaw, Poland

${ }^{*}$ Speaker. 


\section{INTRODUCTION}

Physics has profited in the past from having different types of collisions, which can be generally divided in lepton-lepton, nucleon-lepton and nucleon-nucleon collisions. The LHC has already taken nucleon-nucleon collisions into a new era of energy and luminosity.

The $\mathrm{LHeC}$ intends to make use of the infrastructure of the LHC to take electron-ion collisions into the $\mathrm{TeV}$ era, reaching 400 times the energy achieved by its predecessor HERA, and a luminosity of $10^{33} \mathrm{~cm}^{-2} \mathrm{~s}^{-1}$ or higher with a total power consumption of the lepton facility below 100 MW.

Deep inelastic scattering at the $\mathrm{TeV}$ center of mass $(\mathrm{CM})$ range would complement the studies of the LHC and its discovery potential for physics beyond the Standard Model, as well as providing a unique tool for studying parton structure and precisions Higgs physics.

Five dedicated LHeC workshops have been held between the years 2008 and 2014. The workshop in 2012 was dedicated to the presentation of the Conceptual Design Report (CDR) [?] which included a detailed presentation of the physics, the accelerator options and detector design comprising the $\mathrm{LHeC}$ project.

Two different options for the accelerator were proposed. Firstly, a Ring-Ring (R-R) option, in which leptons are accelerated in a new electron synchrotron storage ring inside the LHC tunnel. Secondly, a Linac-Ring (L-R) option featuring a $60 \mathrm{GeV}$ ERL to accelerate the electron beam. Both designs have been found to be feasible but the R-R option installation would disrupt the LHC operation. Therefore the L-R option has been chosen as the design to follow.

The workshop in 2012 concluded with a CERN mandate to develop the following required R\&D components:

- superconducting RF system;

- superconducting magnet development;

- pipes with large beam acceptance;

- the finalization of the ERL design for the LHeC, including optics, design, beam dynamics studies and identification of potential performance limitations;

- the design and specification of an ERL Test Facility at CERN.

The overall design and the advances on these technical components are presented in this paper.

\section{Energy Recovery Linac and LHeC Test Facility}

The development of a continuous-wave superconducting (CW SC) recirculating ERL chosen as the $\mathrm{LHeC}$ baseline could be benefitial for many possible future projects, e.g., for an International Linear Collider, for a neutrino factory, for a proton-driven plasma wake field accelerator or for a muon collider [?].

The overriding advantage of the ERL baseline design, is that the energy of the spent beam, after collision, is recuperated by returning the beam $180^{\circ}$ out of phase through the same RF structure that had earlier been used for its acceleration, again with several recirculations. The energy recovery efficiency $(\eta)$ reduces the electrical power required for RF power generation at a given beam current by a factor $(1-\eta)$. With an efficiency above $90 \%$ one can reach the beam-current goal of $6.4 \mathrm{~mA}$ with less than $100 \mathrm{MW}$ total electrical power. A schematic view of this ERL is shown in Fig. ??, as 


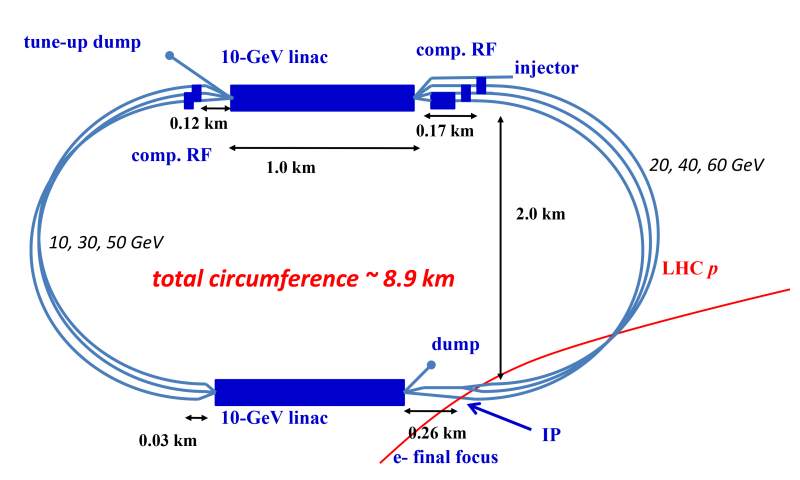

Figure 1: Schematic layout of the $60 \mathrm{GeV}$ ERL option.

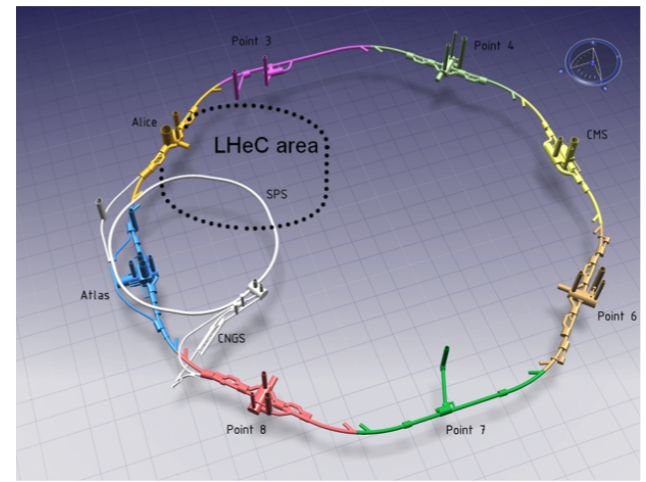

Figure 2: Integration of the ERL into the LHC configuration.

\begin{tabular}{|l|cc|cc|}
\hline & Protons & Electrons & Protons & Electrons \\
\hline Luminosity & $10^{33} \mathrm{~cm}^{-2} \mathrm{~s}^{-1}$ & $10^{33} \mathrm{~cm}^{-2} \mathrm{~s}^{-1}$ & $10^{34} \mathrm{~cm}^{-2} \mathrm{~s}^{-1}$ & $10^{34} \mathrm{~cm}^{-2} \mathrm{~s}^{-1}$ \\
Beam Energy $(\mathrm{GeV})$ & 7000 & 60 & 7000 & 60 \\
Normalized emmitance $\gamma \varepsilon_{x, y}(\mu \mathrm{m})$ & 3.75 & 50 & 2.5 & 20 \\
$\beta^{*}(\mathrm{~m})$ & 0.1 & 0.12 & 0.05 & 0.10 \\
rms Beam size $\sigma_{x, y}(\mu \mathrm{m})$ & 7 & 7 & 4 & 4 \\
rms Beam divergence $\sigma_{x, y}(\mu \mathrm{rad})$ & 70 & 58 & 80 & 40 \\
Beam Current $(\mathrm{mA})$ & $430(860)$ & 6.4 & 1112 & 25 \\
Bunch Spacing $(\mathrm{ns})$ & $25(50)$ & $25(50)$ & 25 & 25 \\
Bunch Population & $1.7 \times 10^{11}$ & $\left(1 \times 10^{9}\right) 2 \times 10^{9}$ & $2.2 \times 10^{11}$ & $4 \times 10^{9}$ \\
Bunch charge $(\mathrm{nC})$ & 27 & $(0.16) 0.32$ & 32 & 0.64 \\
\hline
\end{tabular}

Table 1: L-R LHeC Baseline Parameters

well as the integration of the ERL into the LHC configuration (Fig. ??). The baseline parameters of the design are shown in Table ??.

Several advances have been made towards the design of the LHeC Test Facility (LTF) [?]. Aside from various other technical and physics goals, this test facility will aim to investigate the ERL principle in an LHeC-like configuration, including electron injector and return-arc magnets. It will also provide a test stand for superconducting RF cavity modules, as well as performing LHeCrelated detector R\&D. One possible user application of the LTF beam is for generating controlled beam induced quenches of superconducting magnets.

The LTF is designed to be constructed in stages. A first phase with beam recirculation would only use two 4-cavity cryomodules and single recirculation to reach $150 \mathrm{MeV}$. A second phase could feature multi-pass operation to reach $300 \mathrm{MeV}$ (in 2 passes) or $450 \mathrm{MeV}$ (in 3 passes), adding two more cryomodules could boost the top energy to $900 \mathrm{MeV}$. The final version is shown in Fig. ?? and the baseline parameters are shown in table ??.

Vertical spreaders/combiners separate the beams into up to 3 vertically separated arcs, each of which is optimized for its nominal energy. The highest energy arc is adjusted in length to assure arrival in the decelerating phase when entering the linac again.

The design comprises three different regions, the linac optics, the recirculation optics and 


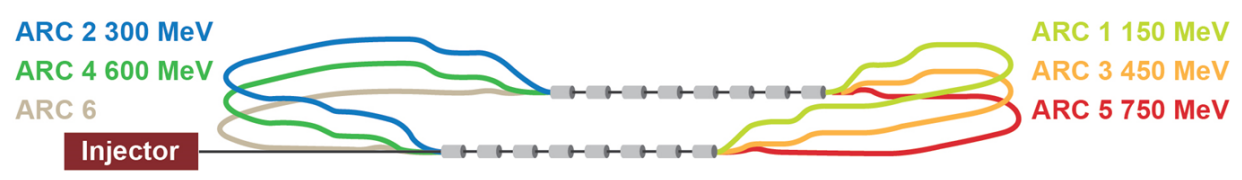

Figure 3: Complete configuration of the LTF with 3 passes of $300 \mathrm{MeV}$ per pass to deliver a $1 \mathrm{GeV}$ electron beam energy.

\begin{tabular}{|c|c|}
\hline Main Parameter & Value \\
\hline Injection Energy $(\mathrm{MeV})$ & 5 \\
Final Beam Energy $(\mathrm{MeV})$ & 900 \\
Normalized emmitance $\gamma \varepsilon_{x, y}(\mu \mathrm{m})$ & 50 \\
Beam Current $(\mathrm{mA})$ & $>10$ \\
Bunch Spacing $(\mathrm{ns})$ & $25(50)$ \\
Acc voltage per cavity $(\mathrm{MV})$ & 18.7 \\
Number of cells per cavity & 5 \\
Cavity Length $(\mathrm{m})$ & 1.2 \\
Number of cavities for cryomodule & 4 \\
Number of cryomodules & 3 \\
RF power for cryomodule $(\mathrm{kW})$ & $<50$ \\
Duty factor & $\mathrm{CW}$ \\
\hline
\end{tabular}

Table 2: LTF Baseline Parameters

the merger optics. The design, at present, is based on a Flexible Momentum Compaction (FMC) cell based lattice. For the optics layout, a possible option would consist of arcs with identical configurations in order to have compact magnets stacked on top of each other.

A complete first-order layout for switchyards, arcs and linac-to-arc matching sections has been accomplished for the arcs on both side of the ERL. The total beam path for a full three pass accelerating cycle is around $280 \mathrm{~m}$. This would require $\sim 200$ magnets.

\section{RF Frequency}

For the SCRF linac system a frequency of $801.58 \mathrm{MHz}$ has been chosen as it is identical to the CERN SPS harmonic system, the LHC harmonic system presently under discussion and it's one of the frequencies envisaged for the Future Circular Colliders study [?]. Strong synergy has been identified with the MESA project at Mainz University; help with the design and construction of the $\sim 802 \mathrm{MHz}$ cavities and cryomodules will result from collaboration with JLAB, who have relevant experience operating CEBAF, and have who have already contributed significantly to the lattice design. 


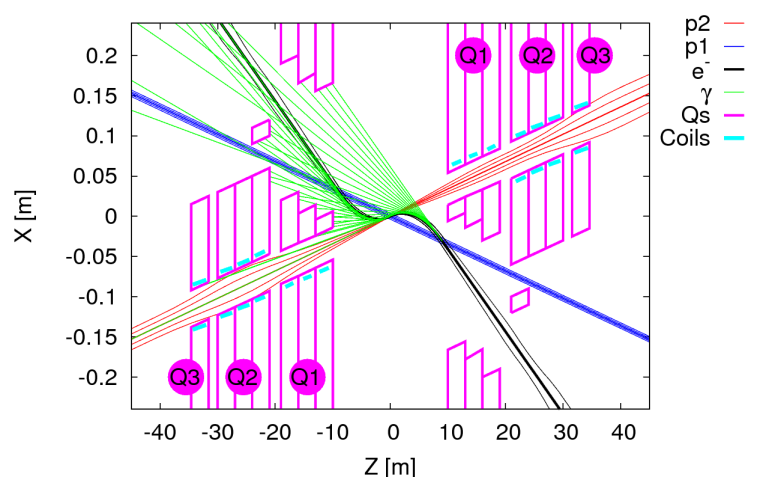

Figure 4: Interaction region schematic view showing the electron beam (black) colliding with the proton beam 2 (red) while the other proton beam (blue) bypasses the interaction [?].

\section{Interaction Region Design}

The interaction region design aims to provide head-on collisions between the proton beam 2 (counterclockwise beam) with the electron beam while proton beam 1 bypasses the interaction. The necessity of increasing the luminosity of the collision calls for a re-designing of the first set of quadrupoles, called the inner triplet, around the interaction point of the proton beam as well as their distance from it. An illustration of the new interaction region design is illustrated in Fig. ??.

The luminosity of the e-p collisions is inversely proportional to the $\beta$ function at the interaction point $\left(\beta^{*}\right)[?]$ :

$$
L=\frac{1}{4 \pi e} \frac{N_{b, p}}{\varepsilon_{p}} \frac{1}{\beta_{p}^{*}} I_{e} H_{h g} H_{D}
$$

In order to achieve the desired luminosity of $10^{33} \mathrm{~cm}^{-2} \mathrm{~s}^{-1}$ the nominal design requires a minimum $\beta^{*}$ of $10 \mathrm{~cm}$, but the limits on the quadrupole strengths and the huge chromatic aberrations caused by the inner triplet means that this desired $\beta^{*}$ cannot be achieved via the normal way of adjusting only the quadrupole strengths. To achieve this, the Achromatic Telescopic Squeezing (ATS) Scheme [?], which has already been studied with great success for the HL-LHC experiment, has been extended to include the $\mathrm{LHeC}$ and reduce the beta function even further in the interaction point and hence increase the luminosity [?].

The flexibility of this design was studied in terms of changing $\beta^{*}$ and $L^{*}$. Minimizing $\beta^{*}$ would increase the luminosity, so the cases $\beta^{*}=5-10 \mathrm{~cm}$ with a fixed $L^{*}=10 \mathrm{~m}$ were found. The case $\beta^{*}=5 \mathrm{~cm}$ is of particular interest since its the value required for this parameter to achieve a luminosity of $10^{34} \mathrm{~cm}^{-2} \mathrm{~s}^{-1}$, as referenced in Table 1 . On the other side, increasing $L^{*}$ minimizes the synchrotron radiation, and the cases with $L^{*}=10-20 \mathrm{~m}$ were found with a fixed $\beta^{*}=10 \mathrm{~cm}$ [?].

The chromatic aberrations are however expected to increase in both previous cases, so the limits of the chromaticity correction have also been explored. The aim is to see if the sextupoles efficiency have been increased enough to fulfill the conditions of stability in the LHC that consists in having an horizontal and vertical chromaticity to a value of 2 and a chromatic amplitude function (Wx and Wy) to a value of 200 in interaction region 3 and interaction region 7, as well as controlling the tune spread on a frequency map. 
The limits of this chromatic correction were found for a maximum $L^{*}$ of $18 \mathrm{~m}$, and a minimum $\beta^{*}$ of $8 \mathrm{~cm}$ [?]. Therefore, the cases $L^{*}=14-18 \mathrm{~m}$ are being considered since they provide the desired luminosity while controlling the chromaticity and also give important benefits in terms of the quadrupole design and the reduction of synchrotron radiation.

\section{CONCLUSION}

The LHeC CDR has been published and describes a nominal design. Several tasks have been addressed since then in order to improve this design. These tasks include the study of the lattice and configuration of the ERL and LTF, the choice of the RF frequency and the interaction region configuration and flexibility. Further development is underway in order to fully validate the design and to explore the feasibility of reaching a luminosity of $10^{34} \mathrm{~cm}^{-2} \mathrm{~s}^{-1}$.

\section{ACKNOWLEDGEMENTS}

Helpful discussion and valuable input from all members of LHeC Study Group Collaboration are thankfully acknowledged. This project has received funding from the European Union Seventh Framework Programme for research, technological development and demonstration under grant agreement no 289485.

\section{References}

[1] J. L. Abelleira Fernandez et al. [LHeC Study Group], "A Large Hadron Electron Collider at CERN" J.Phys.G. 39 (2012) 075001, arXiv:1206.2913.

[2] A.Valloni et al., "Beam physics in future electron hadron colliders", Proceedings of NA-PAC2013, Pasadena, California [TUZAA2]

[3] A. Valloni et al., "Strawman Optics Design for the LHeC ERL Test Facility", Proceedings of IPAC2013, Shanghai, China [TUPME055]

[4] E. Jensen et al., "Design Study of an ERL test facility at CERN", Proceedings of IPAC2014, Dresden, Germany [TUOBA02]

[5] R. Tomás, "Interaction Region" in the Meeting on LHeC with Daresbury group, September 2012. http://indico.cern.ch/conferenceDisplay.py?confId=207665

[6] F. Zimmermann, "LHeC Accelerator Overview" in the LHeC Workshop 2012, Chavannes de Bogis, January 2012.

[7] S. Fartoukh, "An Achromatic Telescopic Squeezing (ATS) Scheme for the LHC upgrade", Proceedings of IPAC2011, San Sebastian, Spain [WEPC037].

[8] M. Korostelev et al., "LHeC IR optics design with integration into the HL-LHC lattice”, Proceedings of IPAC2013, Shangai, China [MOPWO063].

[9] E. Cruz-Alaniz et al., "LHeC IR optics design with integration into the HL-LHC lattice", Proceedings of IPAC2014 [TUPRO070]. 